Case Report

\title{
Airway stenting in a child with spondyloepiphyseal dysplasia congenita: 13-Year survival
}

\author{
Raffaella Nenna, M.D., Ph.D. ${ }^{a}$, , Fabio Midulla, M.D., Ph.D. ${ }^{\text {a }}$, Laura Masi, M.D., Ph.D. ${ }^{\text {, }}$, \\ Giacomo Maria Bacci, M.D. ' , Antonella Frassanito, M.D. a , Roberto Baggi, M.D. ${ }^{\text {d }}$, \\ Maria Luisa Brandi, M.D., Ph.D. e , Stefano Avenali, M.D. ' , Lorenzo Mirabile, M.D. d , \\ Paola Serio, M.D. ${ }^{\mathrm{d}}$ \\ a Department of Pediatrics and Infantile Neuropsychiatry, "Sapienza" University, Rome, Italy \\ ${ }^{\mathrm{b}}$ University Hospital of Florence, AOU-Careggi, Florence, Italy \\ ${ }^{c}$ U.O. Pediatric Ophthalmology, Meyer Children Hospital, Florence, Italy \\ ${ }^{\mathrm{d}}$ Respiratory Endoscopy Unit, Department of Paediatric Anesthesia and Intensive Care, Meyer Children Hospital, Florence, Italy \\ e University of Florence, Florence, Italy
}

\section{A R T I C L E I N F O}

Article history:

Received 26 April 2017

Received in revised form

17 May 2017

Accepted 20 May 2017

Available online 22 May 2017

Keywords:

Airway

Bronchial stents

Pediatric

Spondyloepiphyseal dysplasia congenita

\begin{abstract}
A B S T R A C T
We describe the case of a boy with spondyloepiphyseal dysplasia congenita. At birth, he experienced severe respiratory distress necessitating tracheotomy. Endoscopy done because mechanical ventilation failed to resolve desaturations disclosed severe tracheo-bronchomalacia. A Polyflex silicone stent was placed in the trachea (replaced by Y-Dumon stent) and 2 Palmaz metallic stents in the mainstem bronchi (overlapped with 2 Jomed stents 5 years later). Airway stenting guaranteed a suitable respiratory status and allowed a child who was expected to die at birth, to reach 13.5 years old in good conditions.
\end{abstract}

(C) 2017 Elsevier B.V. All rights reserved.

\section{Introduction}

Skeletal dysplasias belong to a heterogeneous group of disorders involving abnormal bone or cartilage growth or texture [1].

Genetic research has classified several dominant mutations in the type II collagen gene (COL2A1), resulting in skeletal diseases, ranging from lethal dwarfism at birth to relatively mild conditions with little skeletal growth impairment [2].

Within these widely ranging phenotypes, spondyloepiphyseal dysplasia congenita is a disease that presents at birth and has as its hallmark delayed epiphyseal ossification. Infants with this disease are usually premature and stillborn or die shortly after birth from respiratory failure secondary to hypoventilation. When respiratory insufficiency develops in the immediate postnatal life, the only way to achieve a short life after birth is to deliver intensive ventilatory support [3].

\footnotetext{
* Corresponding author. Department of Pediatrics and Infantile Neuropsychiatry, “Sapienza” University Rome, Viale Regina Elena 324, 00161 Rome, Italy.

E-mail address: raffaella.nenna@uniroma1.it (R. Nenna).
}

We described an infant with spondyloepiphyseal dysplasia congenita, in whom prompt airway stenting allowed the boy to reach the age of 13.5 years. The unique clinical and laboratory findings we describe in this teenage boy should help others decide how to care these children.

\section{Case report}

\subsection{History}

The proband was born from unrelated healthy Caucasian parents. He had a maternal half-sister and paternal half-brother. The family history was negative for skeletal dysplasias.

The boy was delivered by emergency caesarian section for fetal distress at 39.5 gestational weeks. The Apgar scores were 6 at $1 \mathrm{~min}$ and 7 at 5 minutes. He weighed $3000 \mathrm{~g}$ (10-25th centile) and he was $47 \mathrm{~cm}$ in length (10th centile). The head circumference measured $39 \mathrm{~cm}$ (>95th centile).

On physical examination the child showed a broad and protruding forehead, large anterior fontanelle $(4 \times 4 \mathrm{~cm})$, short neck, 
short and stocky body with a small rib cage. The child had micrognathia and a wide cleft palate. The abdomen was prominent and arms and legs were unusually short with bilateral clubfeet.

Soon after birth, the infant began to experience severe respiratory distress with repeated obstructive apnea spells, necessitating orotracheal intubation and then tracheostomy. Chest computed tomography $(\mathrm{CT})$ showed a small chest with short ribs and moderately expanded lungs.

Despite high positive end-expiratory pressure $(15 \mathrm{~cm} \mathrm{H} 2 \mathrm{O}$ ) mechanical ventilation by tracheostomy, the child experienced episodes of acute severe respiratory distress. An endoscopic evaluation detected severe complete tracheo-bronchomalacia without signs of external vascular compression. A Polyflex self-expanding silicone stent was placed in the trachea (replaced by a $10 \times 30$ $\mathrm{mm}$ and then by a $12 \times 20 \mathrm{~mm}$ Dumon stent) while 2 metallic expandable stents (Palmaz 104-right and Palmaz 128-left) were placed in the mainstem bronchi and immediately improved the airway lumen. Stents were placed with a rigid bronchoscope (Karl Storz $^{\circledR}$, Germany; $\varnothing 3.5 \mathrm{~mm}$ ) and the patient under general anesthesia (sevoflurane inhalation \pm intravenous propofol) and radiological monitoring, as previously described [4].

The patient's parents signed an informed consent for genetic testing in accordance with regulations of the Institutional Review Board of Florence University Hospital. Molecular analysis disclosed a missense mutation $G>A$ at position 1330 in exon 22 in the COL2A1 gene (c.1330G > A). This substitution changes a glycine at position 313 to a serine (G313S) [2].

Abdominal ultrasonography showed marked colorectal distension. A rectal biopsy obtained during laparotomy for intestinal malrotation excluded Hirschsprung's disease. A percutaneous gastrostomy was packaged and jejunal feeding was started.

Transfontanellar ultrasonography showed congenital triventricular hydrocephalus requiring ventricular drainage at the age of 8 months.

The child was discharged at the age of 18 months with a tracheostomy, tracheal and bronchial stents and continuous ventilatory support, enteral feeding via percutaneous gastrostomy and scheduled for endoscopic follow-up. Oral intake was slowly reestablished one year later.

Unfortunately, the patient was for several years lost to follow-up until the age of 4.3 years, when he was again brought to our attention for respiratory failure and promptly underwent diagnostic bronchoscopy. The trachea appeared almost totally occluded by the broken stent and multiple granulation tissue. The tracheal stent was therefore removed and the ovalized bronchial metallic stents were simultaneously calibrated. Two months later, because of relapsing ovalizations of the bronchial stents causing the loss of adequate radial pressure, two metallic stents (Jomed 12-10/12) were placed to overlap the previous stents in the mainstem bronchi. During a closed endoscopic follow-up bronchial stents were calibrated at the final diameter $(12 \mathrm{~mm})$ and endoscopies scheduled every 6 months confirmed adequate airway patency. At the age of 10.8 years bronchoscopy visualized a severe ovalization involving the stent in the left mainstem bronchus (Fig. 1) and a chest CT scan showed a minute space between the airway and the spine (Fig. 2). A conservative approach with a Y Dumon stent (11-77 replaced by an 11-8-8 stent after 6 months) restored bronchi to their circular shape and lumen patency immediately and markedly improved (Fig. 3A and B).

To date he is a 13.5-year-old child (Fig. 3C), he has a tracheotomy (4.5 mm non-cuffed Shiley with speech valve) and occasionally needs night ventilatory support (99\% oxygen saturation, respiratory rate: 26 breaths per minute and heart rate: 120 beats per minute). The child weighs $15 \mathrm{Kg}$ and measures $84 \mathrm{~cm}$ in length with a head circumference of $56 \mathrm{~cm}$ and a thoracic circumference of $59 \mathrm{~cm}$. Although the child has never been able to walk, a wheelchair guarantees a certain degree of autonomy. He has mild bilateral hearing loss that hearing aids significantly improve. He is only mildly hypermetropic. Baseline serum calcium and 25-hydroxyvitamin $\mathrm{D}\left(25(\mathrm{OH}) \mathrm{D}_{3}\right)$ levels were low and improved after vitamin D supplementation for 2 months. Serum alkaline phosphatase, BAP and $\beta$-CTX were low at baseline and after 2 months. LS-BMD [0.308 $\mathrm{gr} / \mathrm{cm}^{2}$ (LS Z-score: $4.1 \mathrm{SD}$ )] was low at baseline.

He attends secondary school and achieves a fairly good performance.

\section{Discussion}

Our case report describing a boy with spondyloepiphyseal dysplasia congenita related to a G313S mutation who underwent stenting and survived to 13.5 years is distinctly unusual. This mutation has been described only in a child who died of
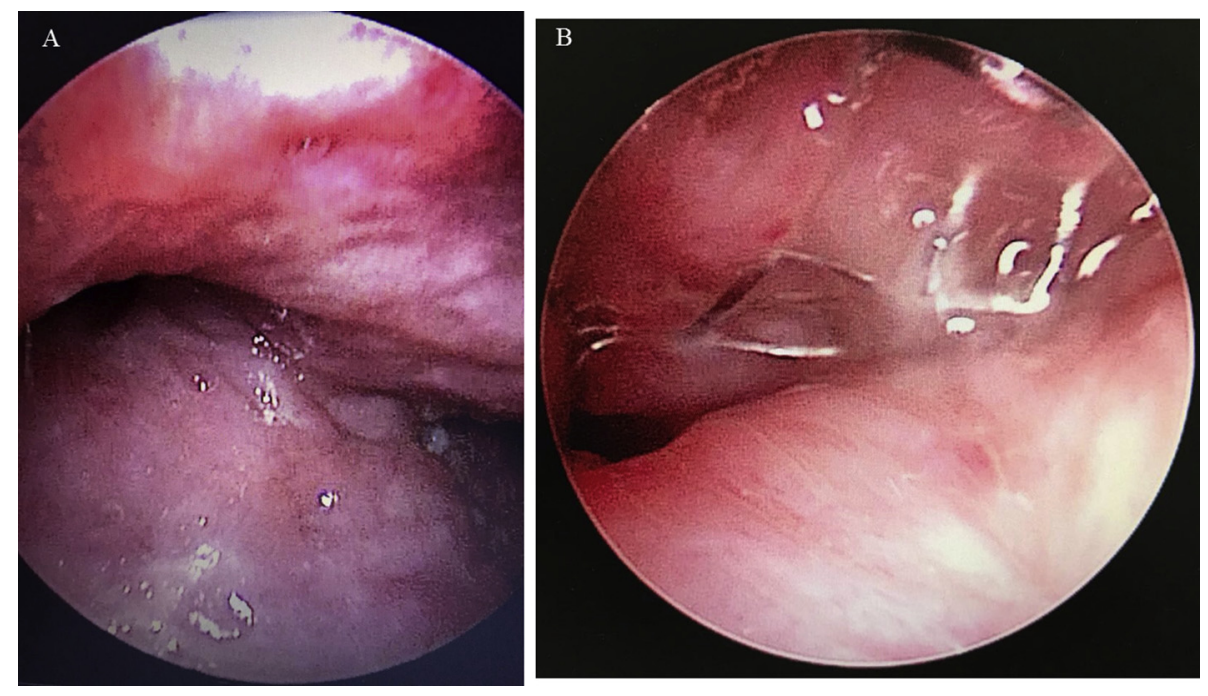

Fig. 1. Endoscopic view of: A) the major carina with partially occluded left mainstem bronchus. B) severe ovalization involving the stent in the left mainstem bronchus. 

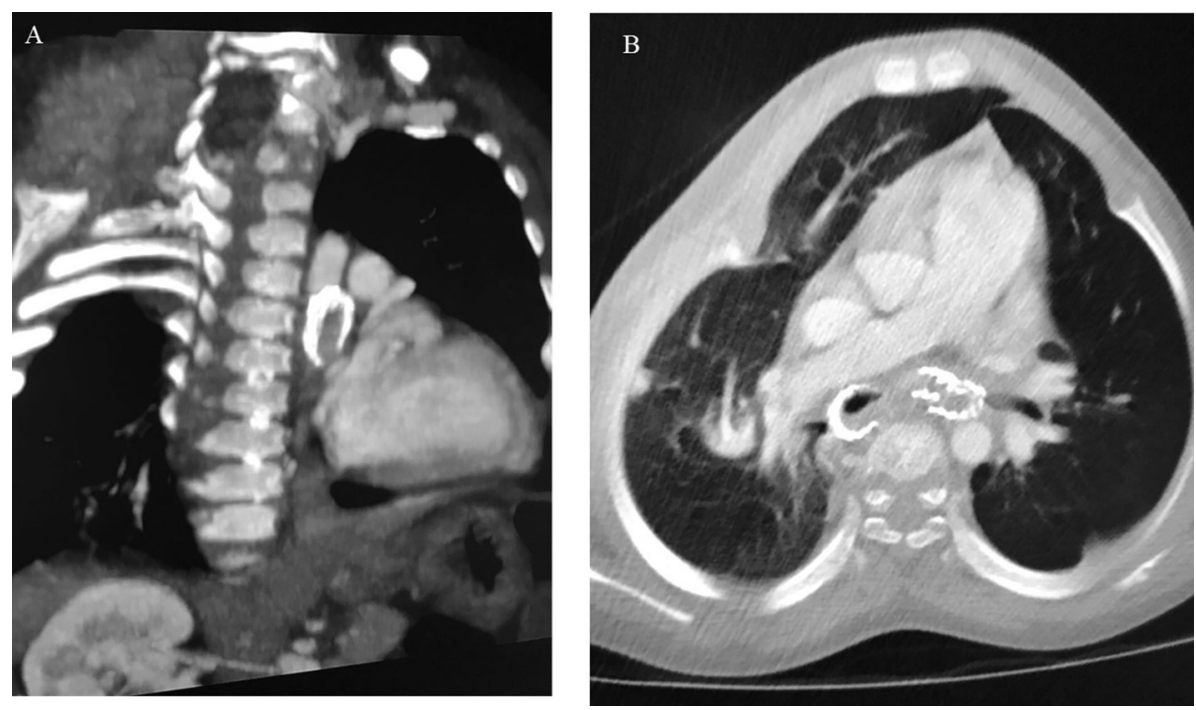

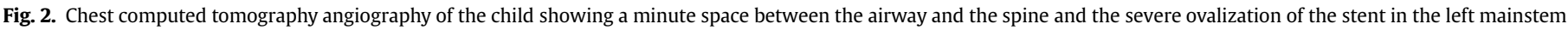
bronchus: A) multiplanar coronal reconstruction; B) axial plane.
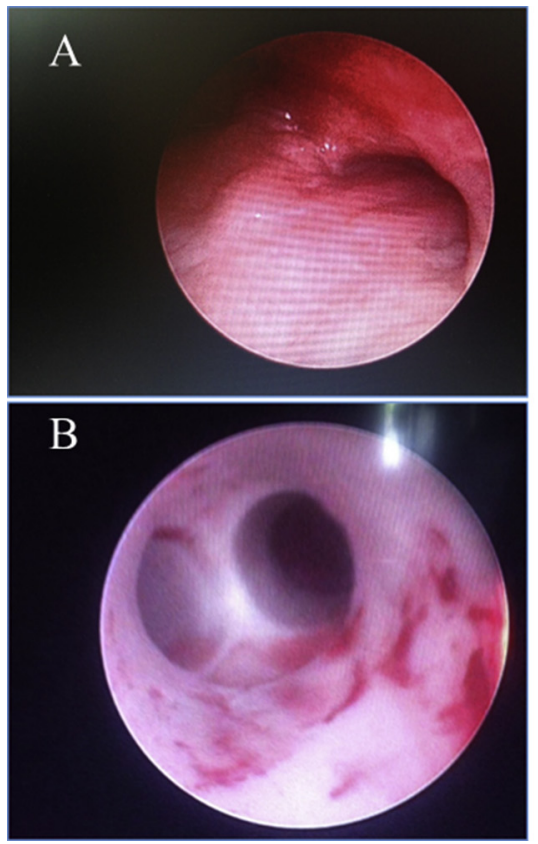

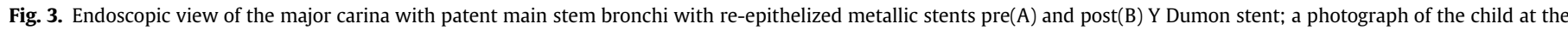
age of 13 years $(C)$.

Legend: Permission was granted by the child's mother to show the face of the patient.

respiratory insufficiency at birth [6]. Mutations in the COL2A1 gene result in widely varying phenotypes depending upon the precise mutation [5].

A feature underlying the importance of stenting in achieving exceptional survival in the child is chronic, severe obstructiverespiratory disease. This ventilatory deficiency arose from several abnormalities including the small rib cage, severe tracheabronchomalacia, hypo-expanded lung and respiratory muscle failure, exacerbated by marked abdominal distention. The prompt stenting corrected the severe airway malacia and improved airway ventilation. Although several authors have described the role of airway stenting in children with severe malacia most of them agree

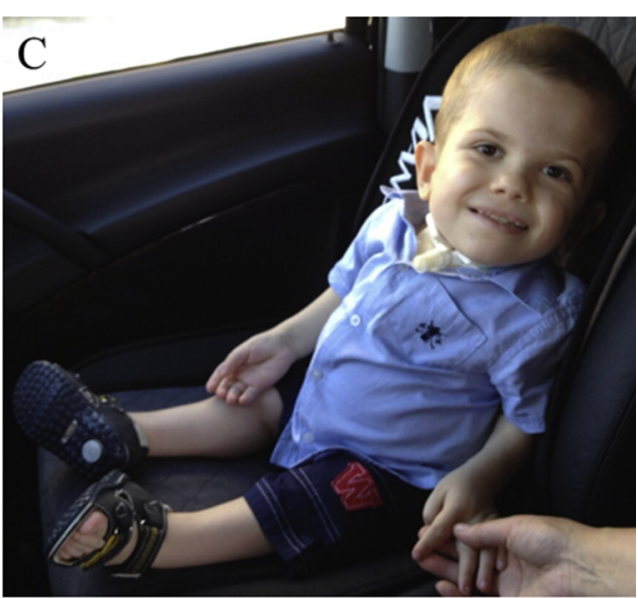

that to avoid possible severe complications stents should be used only as a last resort when no good alternatives exist [6]. Placing a stent in a palliative setting as we did in a child with a poor prognosis related to the underlying disease, is reasonable [7]. Information on tracheobronchial stents in patients with a severely deformed chest is limited to few case reports describing fatal complications, such as fatal tracheal erosion with severe hemoptysis in a child with spondylothoracic dysplasia [8] and an aortobronchial fistula in a patient with severe scoliosis and severe left bronchomalacia [9]. Given that airway stenting is considered hazardous when the reduced anatomical space extrinsically compresses the airway $[8,9]$, this palliative option requires regular, 
watchful follow-up to detect severe complications before they become irreversible.

Although silicon stents can remain intact and last for a long time, when the child returned to our attention after some years, the removable silicon stent was found fragmented into several pieces and almost totally occluding the tracheal lumen. Because it lay over the mucosa rather than being embedded, it was safely removed. Bronchial stents, considered as permanent devices [10], can be adapted to the size of the growing airway by scheduled balloon dilations and easily expanded [4]. After recurrent ovalizations of the bronchial stents due to the small rib cage, a conservative approach with a Y Dumon stent restored bronchi to their circular shape and lumen patency immediately and markedly improved.

Deciding on whether stenting is justified in a newborn child destined to die, is a difficult and controversial problem for physicians to face. We considered conservative treatment with airway stenting as the last therapeutic option in this patient, aimed to "give a chance" to a baby doomed to die but in whom we wanted to palliate the painful respiratory symptoms. The excellent results we achieved surprised us and changed our way of caring for these patients. Knowing more about outcome through managing these patients with airway stenting is the basis for planning up-to-date genetic counseling and perinatal treatment. Until now, the only future predicted for these children has been stillborn or death soon after birth [2].

In conclusion, trachea-bronchial stents allowed this boy with spondyloepiphyseal dysplasia congenita, a genetic disease with a poor outcome, to survive for 13.5 years and attain adequate social inclusion. Others managing this severe disease should find the new clinical information instructive.

\section{Funding}

This research did not receive any specific grant from funding agencies in the public, commercial, or not-for-profit sectors.

\section{References}

[1] A. Panda, S. Gamanagatti, M. Jana, A.K. Gupta, Skeletal dysplasias: a radiographic approach and review of common non-lethal skeletal dysplasias World. J. Radiol. 6 (2014) 808-825.

[2] G.R. Mortier, M. Weis, L. Nuytinck, et al., Report of five novel and one recurrent COL2A1 mutations with analysis of genotype-phenotype correlation in patients with a lethal type II collagen disorder, J. Med. Genet. 37 (2000) 263-271.

[3] L.M. Turner, T.S. Steffensen, J. Leroy, E. Gilbert-Barness, Spondyloepiphyseal dysplasia congenita, Fetal. Pediatr. Pathol. 29 (2010) 57-62.

[4] P. Serio, V. Fainardi, R. Leone, et al., Tracheobronchial obstruction: follow-up study of 100 children treated with airway stenting, Eur, J. Cardiothorac. Surg. 45 (2014) e100-109.

[5] L. Bonafe, V. Cormier-Daire, C. Hall, et al., Nosology and classification of genetic skeletal disorders: 2015 revision, Am. J. Med. Genet. 167A (2015) 2869-2892.

[6] L.A. de Trey, J. Dudley, H. Ismail-Koch, A. Durward, H. Bellsham-Revell, S. Blaney, et al., Treatment of severe tracheobronchomalacia: ten-year experience, Int. J. Pediatr. Otorhinolaryngol. 83 (2016) 57-62.

[7] T. Nicolai, Airway stents in children, Pediatr. Pulmonol. 43 (2008) 330-344.

[8] W.H. Stotz, I.D. Berkowitz, J.C. Hoehner, D.E. Tunkel, Fatal complication from a balloon-expandable tracheal stent in a child: a case report, Pediatr. Crit. Care. Med. 4 (2003) 115-117.

[9] C.H. Cook, N. Bhattacharyya, D.R. King, Aortobronchial fistula after expandable metal stent insertion for pediatric bronchomalacia, J. Pediatr. Surg. 33 (1998) 1306-1308.

[10] P. Serio, R. Nenna, V. Fainardi, et al., Severe residual malacia after surgery for vascular airway compression: treatment with tracheobronchial stenting in children, Eur. J. Cardio. Thor. Surg. 51 (2017) 211-217. 